\title{
Article \\ Investigating Student Engagement and Making Science Real during a Pandemic: Bioskills at Home, a Case Study
}

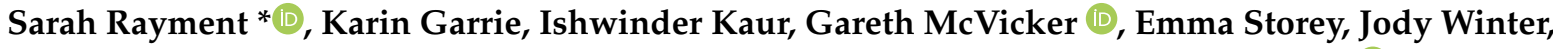 \\ Luigi A. De Girolamo, Callum Rimmer, David Negus, Carl Nelson, Jonathan Thomas (D, Michael Loughlin and \\ Jess Dale
}

\section{check for}

updates

Citation: Rayment, S.; Garrie, K. Kaur, I.; McVicker, G.; Storey, E.; Winter, J.; De Girolamo, L.A.; Rimmer, C.; Negus, D.; Nelson, C.; et al. Investigating Student Engagement and Making Science Real during a Pandemic: Bioskills at Home, a Case Study. Educ. Sci. 2022, 12, 106. https://doi.org/10.3390/ educsci12020106

Academic Editor: Maria Limniou

Received: 12 January 2022

Accepted: 28 January 2022

Published: 3 February 2022

Publisher's Note: MDPI stays neutral with regard to jurisdictional claims in published maps and institutional affiliations.

Copyright: (C) 2022 by the authors. Licensee MDPI, Basel, Switzerland. This article is an open access article distributed under the terms and conditions of the Creative Commons Attribution (CC BY) license (https:// creativecommons.org/licenses/by/ $4.0 /)$.
School of Science and Technology, Nottingham Trent University, Clifton Lane, Nottingham NG11 8NS, UK; karin.garrie@ntu.ac.uk (K.G.); ishwinder.kaur@ntu.ac.uk (I.K.); gareth.mcvicker@ntu.ac.uk (G.M.); emma.storey@ntu.ac.uk (E.S.); jody.winter@ntu.ac.uk (J.W.); luigi.de-girolamo@ntu.ac.uk (L.A.D.G.); callum.rimmer@ntu.ac.uk (C.R.); david.negus@ntu.ac.uk (D.N.); carl.nelson@ntu.ac.uk (C.N.); jonathan.thomas@ntu.ac.uk (J.T.); michael.loughlin@ntu.ac.uk (M.L.); jess.dale@ntu.ac.uk (J.D.)

* Correspondence: sarah.rayment@ntu.ac.uk

\begin{abstract}
Development of key practical skills is fundamental to bioscience courses in higher education. With limitations on access to laboratory time due to the COVID-19 pandemic, a "Bioskills at home" kit was developed to create opportunities for first year undergraduate students to develop these skills using online support resources to guide their activities and build communities of learning. Equipment and activities in this kit enabled students to practice key skills such as pipetting, data handling, experimental design and microscopy, as well as build an online peer learning community through the use of discussion boards and microscopy competitions that encouraged students to explore their local environment. Students who engaged with these activities reported increased confidence in key practical skills. Practical assessment of skills showed that that there was no reduction in the proportion of students who succeeded in achieving the pipetting learning objective compared to previous years, despite a significantly reduced on-campus provision. Although the celebration event to choose the microscopy competition winners was well attended, there was limited use of the discussion boards by students to build a community of learning during the term. Refinement of this initiative will focus on providing greater scaffolding to encourage greater engagement with activities and enhance community building.
\end{abstract}

Keywords: bioscience; home labs; COVID-19; practical skills development; learning communities

\section{Introduction}

\subsection{Practical Science and Meaningful Learning}

Practical classes are a key aspect of teaching in science as they provide the opportunity for students to experience theoretical concepts in a real-world environment [1]. Such experiences are beneficial in fostering interest in the subject and motivating students, both of which have long been recognised as important factors in student learning in science. Studies in secondary schools highlighted that even sixth form (post-16 education) students (who had been considered to have demonstrated an interest in science through their choice of study) reported that laboratory classes increased their enjoyment and interest in their subject [2]. A recent study further highlighted that university students found that student motivation was increased further if they were able to conduct laboratory-based research (open-ended enquiry) as part of their course [3].

Learning theories, such as Novak's model of meaningful learning [4], support the idea that practical work would enable students to integrate their experiences to create a deeper learning experience. This theory, which evolved from the concepts described in Ausubel's assimilation theory for cognitive learning [5], described that for individuals to form a network of interconnected long-term memories (rather than rote learning) requires 
integration of new ideas or information with their pre-existing knowledge. According to Novak's model, to achieve this required both cognitive (reasoning) and affective functions (such as student interest/motivation). More recently, it has been recognised that meaningful learning in a laboratory setting requires not only these domains but also psychomotor functions such as precision [6]. The model of meaningful learning has been examined through the development of the Meaningful Learning in the Laboratory Instrument (MLLI) which has shown the importance of all three domains in student learning $[7,8]$.

It is widely accepted that enquiry-based learning has positive benefits in terms of fostering deeper levels of learning and building students' transferrable skills [9,10]. Studies at a secondary school level show that enquiry-based learning in laboratories develops student metacognitive skills as they have control over problem solving and subsequently build awareness of their own physical and cognitive skills [11]. In this context, development of metacognitive skills would not only lead to more meaningful learning but also enable students to apply these problem-solving skills to other areas of their academic and personal life.

A review of literature relating to university laboratory provision also described how using open-ended enquiry-based laboratories improved learning outcomes as well as being more enjoyable [12]. Examples of this can be seen throughout bioscience disciplines such as the RNA interference experiments described by Kudell [13], contextualisation of investigations into disease-causing proteins using authentic medical case files [14] and development of a programme for students to work together in small groups on exercise physiology projects [15].

However, a report based on a discussion between bioscience academics recognised student interest was only one of several potential benefits of practical classes; they also highlighted the importance of practical classes in developing practical skills, confidence building, awareness of lab health and safety, better understanding of how scientists approach addressing research questions, as well as helping to foster a sense of professional identity [16]. Similar observations about the importance of building practical competencies have been explored in chemistry $[17,18]$.

\subsection{Institutional Context}

The onset of the current COVID-19 pandemic has presented a significant challenge when planning for delivery of the twelve Royal Society of Biology accredited practical skills-based biosciences courses at our institution. Our undergraduate degree programs usually span three years, with all full-time courses offering the option to additionally carry out an industrial placement ("sandwich") year if students aspire to gain work experience in a workplace setting. Each degree program is comprised of equally weighted modules, some of which are taken by more than one degree program. For example, students on all degree programmes will undertake the "Practical Techniques in Biology" module.

Plans for the 2020-2021 academic year required a balance between our professional body and associated accreditation requirements, the subject benchmark standards as described by the Quality Assurance Agency [19], and occupancy restrictions that were imposed to create a flexible COVID-secure learning and teaching environment. All of this took place amidst changing government guidelines due to emerging scientific discoveries about the nature and transmissibility of the virus [20].

All our bioscience courses have a central ethos of providing quality, work-place-like practical classes linked to course disciplines in our state-of-the-art laboratories [21]. In the 2014 audit of provision of "wet" laboratory opportunities for first year and second year bioscience students across the UK higher education sector, it was reported that students received an average of $98 \mathrm{~h}( \pm 39 \mathrm{~h}$ ) of laboratory time [22]. At that time, our bioscience courses provided above average provision of wet laboratory classes $(108 \mathrm{~h})$. However, it should be noted that this has reduced in the intervening years with the increased availability and implementation of dry laboratory options. The COVID-19 pandemic required a compromise in the offering and reduction in the number of opportunities to practice lab skills due to the reduced laboratory occupancy levels mentioned above. 
To facilitate the rationalisation of practical session provision, laboratory classes were mapped to learning outcomes and skill sets that students in each course were able to acquire at each level, using a skills tracker that has been developed within the department (see Supplementary Materials, Table S1). A key consideration in this was the expected graduate attributes that were set out by the Royal Society of Biology degree accreditation programme [23]. We also considered which level of the course students were at during the 2020-2021 academic year and which skill sets they already had acquired earlier on in the course. As such, we identified our first-year students, who had already been affected at college or school in 2019-2020 before joining us, as a year group that may benefit most from additional opportunities to gain confidence and familiarity with scientific equipment and procedures while being at home as well as benefitting from more opportunities for community building and peer-to-peer interactions. This level also presented the simplest labs to mimic safely in the home setting.

\subsection{Supporting Practical Science Outside the Laboratory}

As technology has advanced, the range of methods that academics have available to develop students' understanding of the links between their theoretical and practical work has expanded. In a similar way to practical laboratory experiments, simulated laboratories can have a significant impact on student understanding of processes and real-world phenomena [9]. In addition, creating virtual learning environments (VLE), such as that described by Sotomayor-Moriano et al. [24], allows students to explore aspects of their subject which might otherwise be inaccessible to them. In the scenario described by Sotomayor-Moriano et al., this refers to access to equipment but can also refer to health and safety or ethical concerns that would make the experiment prohibitive (examples are described by Lewis [25]). These virtual enquiry-based laboratory experiences have been described as being as effective as using protocol-driven practical laboratories in terms of student learning, at least as measured by exam performance [26].

A further consideration in the use of resources and simulations is that they can be used alongside laboratory classes (rather than replacing them) to scaffold student learning or skill development. Studies have shown that familiarising undergraduates with material related to their class before the class itself (e.g., using web-based activities and quizzes) increased lab learning gains as measured by the proportion of students who could achieve one of the lab learning outcomes on their first attempt (plating bacteria for single colonies; [27]), as well as having the potential to increase student confidence $[28,29]$ and feelings of preparedness [30].

In response to the COVID-19 pandemic, bioscience academics from numerous universities have created a community of practice called DryLabsRealScience designed to provide support in developing alternatives to wet lab classes and capstone projects [31]. In addition to sharing practice, this group also curates resources that are hosted on the lectuREmotely webpage: this website was part of an initiative by colleagues at De Montfort University to support staff in developing teaching strategies and accessing resources during the pandemic [32].

However, whilst these technologies can have significant benefits, it is important to recognise their limitations: simulations and other resources are limited by their inability to provide hands-on training in the use of individual techniques or pieces of equipment, which is an important aspect of student learning in bioscience disciplines [25]. In the context of this study, for example, virtual resources could be used to support familiarisation with correct pipetting, but it would not prepare students for the experience of performing the pipetting action and identifying the change in resistance at the stopping points. Additionally, the data generated using these models are limited by the assumptions used to create them, which means that in most cases students do not experience atypical data, as can be the case in laboratory experiments. Arguably, students benefit from these experiences as it enhances their problem-solving skills, confidence and resilience in dealing with failed experiments and, potentially, their understanding of the theoretical concepts underpinning 
the techniques they are using. In particular, purposefully using a productive failure design as that used by Lam [33] can allow students to learn more deeply than if they initially succeed. This cognitive processing used questions and generation of ideas and explanations to address experimental observations, although, in this study, this learning was a collaborative process that brought students to consensus through constructive argument which could be later consolidated by staff.

\subsection{Community Building}

Another aspect that has been disproportionally affected by the reduction in practical sessions is the social aspect of the laboratory class. Feeling a sense of connection to their course and peers may be particularly problematic for this year group as they have not made the choice to learn remotely and have not already built a network of peers through previous years of university-level study.

Research from as far back as 1963 has shown that students appreciate the opportunity to learn in an environment in which there is social interaction between themselves, their peers and educators [2]. Indeed, the idea that peers can influence student learning is a concept that has its roots in Vygotsky's social constructivism [34]. In addition, working in small groups in the laboratory has also been shown to help students develop a sense of social cohesion, which leads to a more collaborative and effective learning environment [9].

Learners who are studying remotely often feel a sense of isolation and loneliness, and this negatively impacts both on their learning and retention [35-37]. A key aim of building learning communities is to help these first-year students who have yet to develop a network of peers to feel connected to their course and their peers. These interactions, which can be peer-peer or peer-academic, help to develop students' understanding and sense of belonging, which will support their learning, attainment and progression.

\subsection{Aim}

The aim of this research was to develop a program of experiments for first year undergraduates to be able to undertake in their home environment to build confidence in key experimental skills and facilitate interaction between students. For first year students who had not had the opportunity to form social networks prior to the COVID-19 pandemic and were going to now receive a blended approach to learning with limited opportunities for face-to-face learning, the development of forum discussion around the activities was considered an important aspect. It was hoped that this initiative would foster a sense of community amongst students as well as build student confidence and competence in key academic skills.

\section{Materials and Methods}

\subsection{Planning the Bioskills at Home Experiments}

All bioscience students are required to evidence competency in key laboratory skills in their first year, namely the accurate use of micropipettes and ability to light microscopy for cell counting. This is an on-campus assessment that takes places at the end of this first term after the laboratory sessions have been completed.

In term 1, the number of on-campus laboratory sessions was reduced from six to two so when considering how to align the home experiments, ensuring the students had opportunities to practice assessed skills was a key consideration. A review of the six laboratory sessions that would normally take place in term 1 allowed identification of some that could be easily and safely adapted to be carried out by potential novices at home whilst remaining aligned with the teaching content and learning outcomes (LO). The skills tracker (as outlined in the Supplementary Materials) was used to identify what opportunities for skill development would be "lost" due to a number of first year lab classes not taking place in the 2020-2021 academic year, and these were mapped to ensure there were alternative opportunities available in other years of the course. Where possible, we aimed to further support the development of these skills by their inclusion in the home lab experiments. 
In addition to ensuring that home labs were aligned with learning outcomes for the term 1 modules and that they supported development of practical skills, when compiling the list of kit contents, it was considered imperative to minimise the risk of barriers to participation. For this reason, students were provided with everything required, with the exception of the food substances used in the yeasts' growth medium. The pack contained an introductory leaflet which explained what the pack was for, to take care and only use the kit as directed (for safety reasons) and where to access activity instructions; these instructions were hosted through the institution's VLE. Staff were able to monitor engagement with the activities through interaction with these resources or the discussion boards.

\subsection{Pipetting}

Accurate pipetting is a core bioscience skill that underpins a wide range of techniques and assays that are used extensively throughout undergraduate courses and one that our institution assesses in person at the end of the first term. This assessment usually takes place once students have completed their six term one practical classes, which offer multiple opportunities to practice the skill. However, in 2020/2021, the number of oncampus laboratory opportunities to familiarise themselves with the equipment and process of pipetting prior to assessment were reduced to two due to social distancing. Providing students with opportunities to build confidence and reproducibility in this technique through bespoke exercises using the Bioskills at home kits was therefore identified as a priority. These activities were specifically designed for this initiative, as the development of pipetting skills is integrated into the range of practical classes undertaken by students throughout term 1, rather than being taught separately. Each student was provided with the three micropipettes most commonly encountered in undergraduate practical classes: 2-20, 20-200 and 100-1000 $\mu \mathrm{L}$ (see Figure 1).

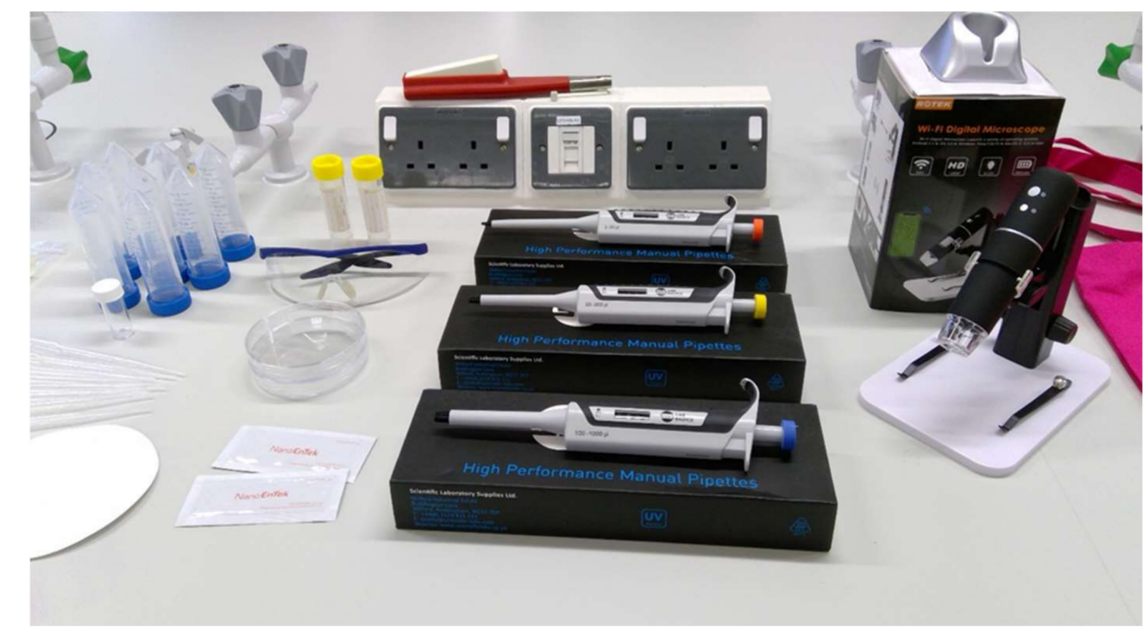

Figure 1. Essential equipment and consumables that formed the basis of the Bioskills at home kit including micropipettes of three assorted sizes (centre) and a digital microscope.

The activities designed for this section of the Bioskills at home initiative were aimed at supporting the students with handling the micropipettes, guiding them with loading and emptying. Practicing the pipetting action was considered a low-risk activity as it did not always require the use of liquid and for most activities the students could practice using water. A suite of resources was created in the university VLE which incorporated video resources tailored to each activity, written guidance and pre-lab simulations from Learning Science (Bristol, UK) for using different types of pipettes.

The pipetting exercises using 2-20, 20-200 and 100-1000 $\mu \mathrm{L}$ micropipettes were based on the 2,3,5 exercises described by Professor Wolf [38]. The dilution exercise was also developed to aid students in preparing 1 in 2, 1 in 5 and 1 in 10 dilutions using all three volume ranges of micropipettes mentioned above. 
Further exercises supported students in developing the ability to check the accuracy and precision of their pipetting skills, as well as troubleshooting issues with pipetting. These experiments gave the students measurable results that they could analyse to selfassess the reproducibility of their pipetting and therefore continue to improve their skills. To accomplish this required the students to use a coloured liquid that could be pipetted onto filter paper and zones of spread measured. It was important to minimise the safety risk and so the coloured solution supplied in the Bioskills kit was a domestic food safe colourant (a blackcurrant cordial concentrate). The final iteration of resources for this activity consisted of introductory material and bespoke videos introducing students to the activities, as well as a targeted video for each of the 4 tasks.

\subsection{Microbial Growth}

In the first term, students would usually construct a yeast growth curve for Saccharomyces cerevisiae var. carlsbergensis in the laboratory by measuring changes in optical density (resulting from yeast growth) of growth media using a spectrophotometer. The aims of this experiment are to aid their understanding of associated lecture content about the rate of microbial growth compared to higher organisms, gain experience in plotting graphs (using a semi-log scale) and develop skills in data analysis. This experiment also supported concepts explored in assessed coursework undertaken as part of the microbiology module (a module taken by many students in their second term); students could use data from their home experiment for this coursework if they chose to.

Due to the need to rationalise the provision of laboratories, students did not complete this experiment in class. However, given that the learning objectives associated with this practical were integrated both within and between modules, this experiment was identified as one that would have value in the Bioskills at home initiative.

There were a number of logistical and safety issues that needed to be overcome to ensure the learning objectives could be met in a home environment. Perhaps the most obvious of these was that the students would not have access to a spectrophotometer. As an alternative to this, students were provided with a fading greyscale number scale (as shown in Figure 2) and were asked to record the highest number that they could observe through their experimental tube. Importantly, working with the scale rather than the spectrophotometer allowed the experiment to be re-designed to incorporate an additional learning objective and was a good way to prepare students who may use McFarland standards (which is widely used in antibiotic susceptibility testing; [39]) later in their course. This learning objective was that students should understand the importance of including controls in the experimental design. In the Bioskills at home version of this experiment, students set up a non-inoculated control to show that the media they had created at home was not contaminated.

Another logistical issue was that it was not possible to provide students with individually prepared tubes of pre-sterilised media for their experiments so it was necessary to consider how students could create a suitable media in a home environment. An important consideration was to ensure that the uninoculated media was clear so that the scale could be read throughout. To achieve this, several different products were tested but Marmite (Unilever, London, UK) mixed into hot water was selected as the basic growth media (see Figure 2). In the development process, it was observed that there was a difference in the growth of the yeast in the Marmite media with or without granulated sugar (freely available in shops) dissolved into the Marmite media (1 heaped teaspoon for each $240 \mathrm{~mL}$ of media). Comparison of microbial growth in the presence or absence of sugar formed the primary experiment that students conducted. This experiment, in itself, allowed students to consider what factors could influence the growth of micro-organisms and why this was the case. Students were encouraged to extend this to consider the impact of other substances found in their home environment on microbial growth. For example, how was growth affected by products with potential for anti-microbial properties such as mouthwash? 
A

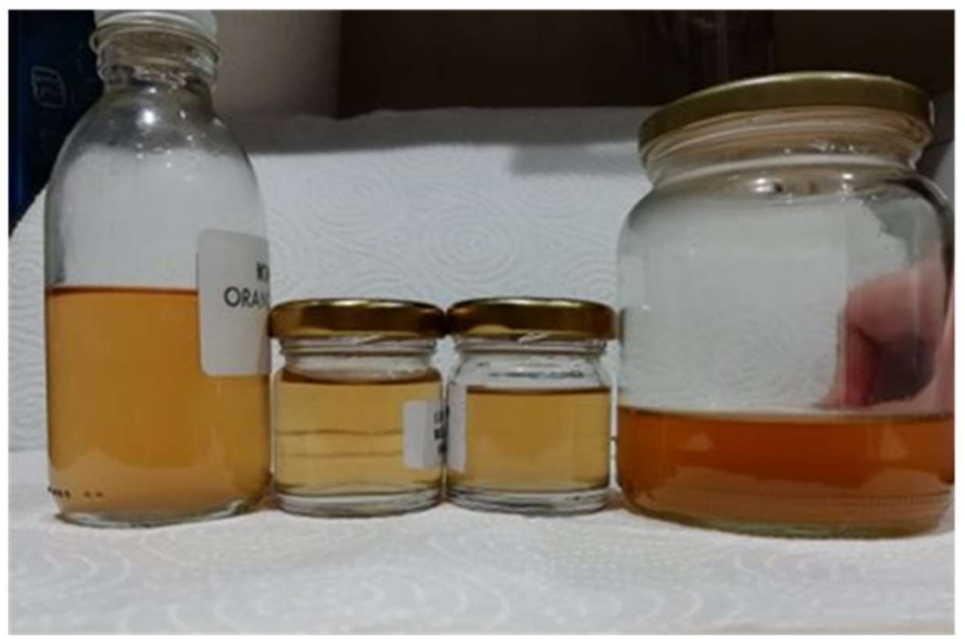

B

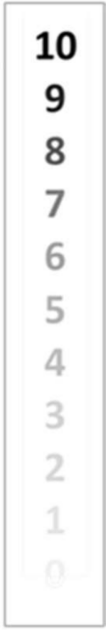

Figure 2. Image (A) shows examples of growth ( $24 \mathrm{~h}$ ) in control and inoculated samples, in a range of different media created to test which would be the most suitable for student use. From left to right, these were: apple juice (inoculated with yeast); Marmite/sugar media (uninoculated control); Marmite/sugar media (inoculated with yeast); Marmite/sugar media (inoculated with soil). Note the difference in opacity between the middle two samples, indicated by the visibility of a metal skewer held behind them. This concept evolved into a number scale used in the final version (which is shown in image (B)).

There were a number of health and safety issues that needed to be addressed for this experiment to be suitable for the home environment. These are outlined in Table 1, alongside the steps taken to address them.

Table 1. Summary of the health and safety considerations for the Bioskills at home microbial growth experiment.

\begin{tabular}{cc}
\hline Issue & Resolution \\
\hline Use of micro-organisms & $\begin{array}{c}\text { Yeast used was changed to dried baker's yeast that is considered } \\
\text { "food grade" and stable at room temperature until use. } \\
\text { Students were reminded to never ingest any of the experimental } \\
\text { components and to wash hands afterwards to minimise the risk } \\
\text { of contaminating their food preparation area. }\end{array}$ \\
\hline $\begin{array}{c}\text { Proximity of water and } \\
\text { electricity/use of boiling } \\
\text { water }\end{array}$ & $\begin{array}{r}\text { Students were reminded of the need to take care when using } \\
\text { water and electricity in close proximity. }\end{array}$ \\
\hline Disposal of microbial cultures & $\begin{array}{c}\text { Students were informed of correct disposal methods including } \\
\text { the need to loosen lids prior to disposing of the cultures due to } \\
\text { potential gas build up. }\end{array}$ \\
\hline
\end{tabular}

\subsection{Microscopy}

Under normal circumstances, students would have multiple opportunities to use a binocular compound microscope to visualise a range of samples, as well as a practical-based seminar that is dedicated to microscope alignment and focussing. Students' opportunities to practice with the compound microscope were limited to one session (instead of three) due to the availability of lab time during the COVID-19 pandemic. The light microscopes used in the teaching laboratories can reach a magnification of $1000 \times$ (using the $100 \times$ oil immersion lens), which is suitable for observing bacteria. However, they are large, heavy and expensive, making them unsuitable for a home lab experience.

Knowing that it would not be possible to replicate the microscope experience that students would have had in the laboratory, the aim of including this type of experiment in 
the Bioskills at home pack was to focus on the other ways in which this type of skill could be used. An important consideration for selecting an appropriate microscope for the students to use was its ability to capture images and short videos. The intention for the first term microscopy activities was to stimulate the students' scientific interest in the world around them, as well as to build engagement with the university's VLE (and each other) through a series of microscopy competitions. This was a valuable opportunity for community building, which was otherwise likely to be limited because of restrictions in place due to the pandemic. Competitions were run for the first two months of the first term with students asked to submit their best images and videos to the following competition categories:

1. A "Tardigrade hunt", where students were challenged to find a tardigrade (also known as a "water bear"; it is an eight-legged invertebrate approximately $1 \mathrm{~mm}$ in length that can be found in damp moss) having been given details of their biology, where they could be found and example videos of how they might appear under a microscope.

2. Best biological image: themes to explore were plant life, tiny creatures, cells and tissues and unusual perspectives on living things.

3. Best biological video: themes were pond life, findings in soil or mud, surprise or funny events captured.

4. Mystery images: students were asked to present a magnified image for others to guess what was being shown.

Students were given the opportunity to vote for competition prize winners (awarded a certificate and prize) during an online celebration event at the end of the first term. This included the mystery images competition where students made their guesses of what the magnified entries were during the session. Each student who contributed a mystery image received a certificate and a prize, as did the person who correctly guessed what the image was.

For students to be able to participate, the microscope that they were provided with needed to be capable of sufficient magnification to be able to view cells, protists and other biologically interesting specimens (e.g., tardigrades). They also needed to be affordable, compact, lightweight and compatible with a range of devices, including smartphones and laptops so that all students would have an opportunity to participate, irrespective of whether they had regular access to a computer. Academic staff tested the small Rotek-EU digital USB/WIFI microscopes (Shenzhen, China; as seen in Figure 1) at home during the first pandemic lockdown in spring/summer 2020 and were able to view a range of different specimens of appropriate size as can be seen in Figure 3.

Students were provided with a written guide on the VLE to ensure that they were supported in their use of the equipment, with additional opportunities for discussion of any issues encountered via the discussion forum. Protocols for microscopy were risk assessed for the home environment prior to assembling the student kits.

\subsection{Delivering the Bioskills at Home Kit to Students}

Based on the experiments that the academic team had designed, a list of kit components was drawn up. These elements all needed to be costed, sourced and delivered within the timescale and within the financial constraints of the project. A bespoke bag was designed and sourced to provide a way for all these components to be safely packaged and easily transported home by students. The estimated cost of the kit only was GBP 100 per student.

As can be seen in Figure 4, the components of the kit included: a range of plasticware (including multiple sizes of pipette tips, tubes of diverse sizes and weighing boats) that was available within the university laboratories but needed to be individually packaged, reagents which needed to be aliquoted and labelled, individually printed guidance on safe use of the kit and a printed scale for the microbiology experiment and health and safety equipment. Overall, 1000 Petri dishes, 2000 microscope slides, 2000 coverslips, $400050 \mathrm{~mL}$ tubes, 5000 plastic Pasteur pipettes, 6000 gloves, 11,000 microcentrifuge tubes and 40,000 pipette tips had to be counted and bagged. 


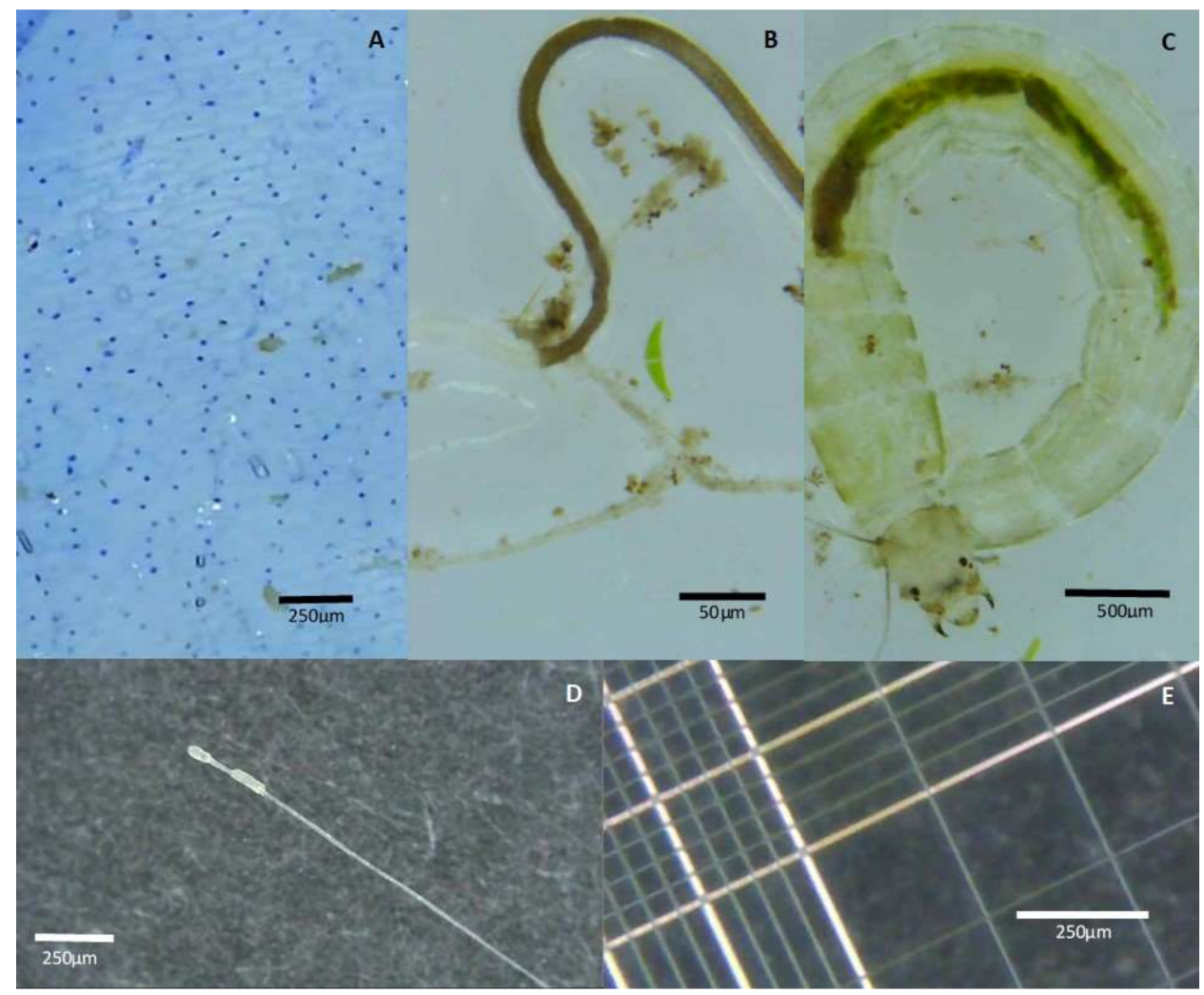

Figure 3. Sample of microscopy images produced by academic staff using the Rotek digital microscope. Images show onion skin stained with ink with visible cell walls and dark blue staining of nuclei (A), nematode worm and Closterium (desmid, freshwater algae) (B), chironomid (non-biting midge larva (C), a human hair (D) and a C-chip haemocytometer (E).

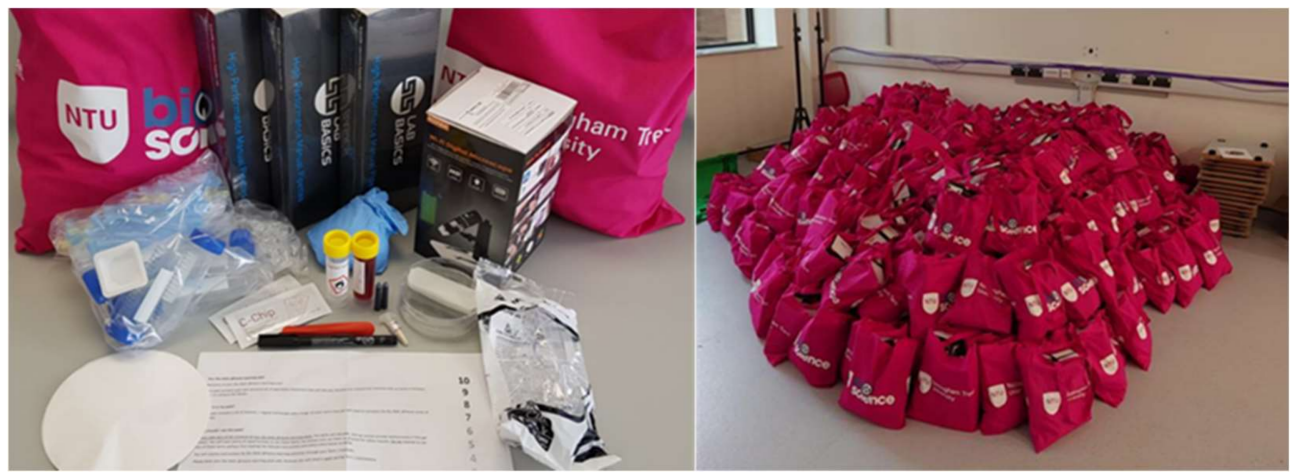

Figure 4. Example of a deconstructed term 1 Bioskills at Home kit including kit bag, protocol, consumables, pipettes and microscope (left) and the completed collection of bags prior to distribution to students (right). [Images have been obscured with white boxes for anonymization purposes].

There were several significant challenges to overcome in the successful delivery of the Bioskills at home kits. First, the number of students in the 2020-2021 intake was significantly larger than had been predicted (an increase of $20 \%$ on the previous year), meaning that the required number of pieces of equipment rose significantly and that the time taken to put the kits together also increased substantially. It took approximately $200 \mathrm{~h}$ (spread across the technical team) to put the kits together, time which needed to be balanced against the existing commitments of preparing for a new academic year and the increased time-pressure resulting from adjustments necessary to ensure that teaching labs were COVID-secure. The impact of the pandemic itself in complicating this process cannot be underestimated, as much of the kit preparation was conducted by team members working from home, with the 
requisite requirement for transporting kit contents between campus and home. This presented significant logistical challenges and required a high degree of co-ordination and planning between team members to effectively deliver the project.

\subsection{Logistics of Distributing Bioskills Packs to Students}

In the first week of term, students had the opportunity to meet with their personal tutors and fellow students in their tutor groups. Being one of only a few sessions that students would have face-to-face in the first term, this was an ideal opportunity to distribute the Bioskills bags. A room was secured to act as the base for storing the completed Bioskills bags and tutors were asked to collect bags for their groups from this base (as shown in Figure 4). This was thought to be the most efficient and COVID-secure way to deliver the bags as tutorial sessions took place at different times across campus. However, it was soon recognised that many students were not able to collect their bags as they were either ill with COVID-19, self-isolating or unable to come to campus due to either personal reasons or travel restrictions.

Students who were later able to attend campus for lab classes were able to collect the kits at the end of their lab sessions or from campus-based drop-in sessions run by the technical team. Unfortunately, not all students were able to collect kits in person, so further support from the technical team and the school's administrative team was necessary to ensure delivery of the Bioskills kits to these students. The first step was to ensure that all kit components could be safely delivered through the postal system, including whether they could be sent internationally. This required talking to multiple couriers as destinations ranged across several continents, including UK and mainland Europe, South America, Africa and Asia. Although students received their kits at different times, the timing of the activities was flexible with the only deadline being for the microscopy competitions, which closed at the end of November.

Given the logistical challenge of decontaminating equipment and the benefit of easy access to equipment, such as micropipettes, throughout their degree, Bioskills bags were not returned at the end of the year.

\section{Results}

The Bioskills at home initiative packs were successfully delivered to all first-year students in term 1 of the 2020-2021 academic year either during tutorial sessions, oncampus drop-in sessions or via delivery to their home address (where necessary). Of the prospective intake of 507 students, a total of 450 students were enrolled into the term 1 modules. Engagement with individual activities is described below but student reflection on the experience of using the kit is highlighted in the quote below:

"I personally found the home kits to be a challenging and engaging way to improve my practical skills in a way that positively impacted my performance in lab assessments".

\subsection{Pipetting Skills}

An example of the expected outcome for the 4th task set for the students can be seen in Figure 5. Whilst discussion about the pipetting activities was very limited on the discussion boards, the proportion of students who accessed resources for the various activities on the VLE was much higher.

Based on the VLE analytics, $43.8 \%$ of students engaged with the introduction to the learning pack and $22.9 \%$ of students engaged with the pipette home exercise guidance online. The videos that were created to support the pipetting activities were watched by $12.7-19.6 \%$ of students with the video for the first exercise (how to operate the pipettes to dispense liquid) the most watched. Learning science resources were engaged with by $7.1-10.0 \%$ of students. With the exception of the introductory material, these analytics showed a lower proportion of student engagement than other formative activities such as pre-recorded lectures, seminars and laboratory protocols (31.4-90.3\%) and quizzes (26.8-37.4\%). 


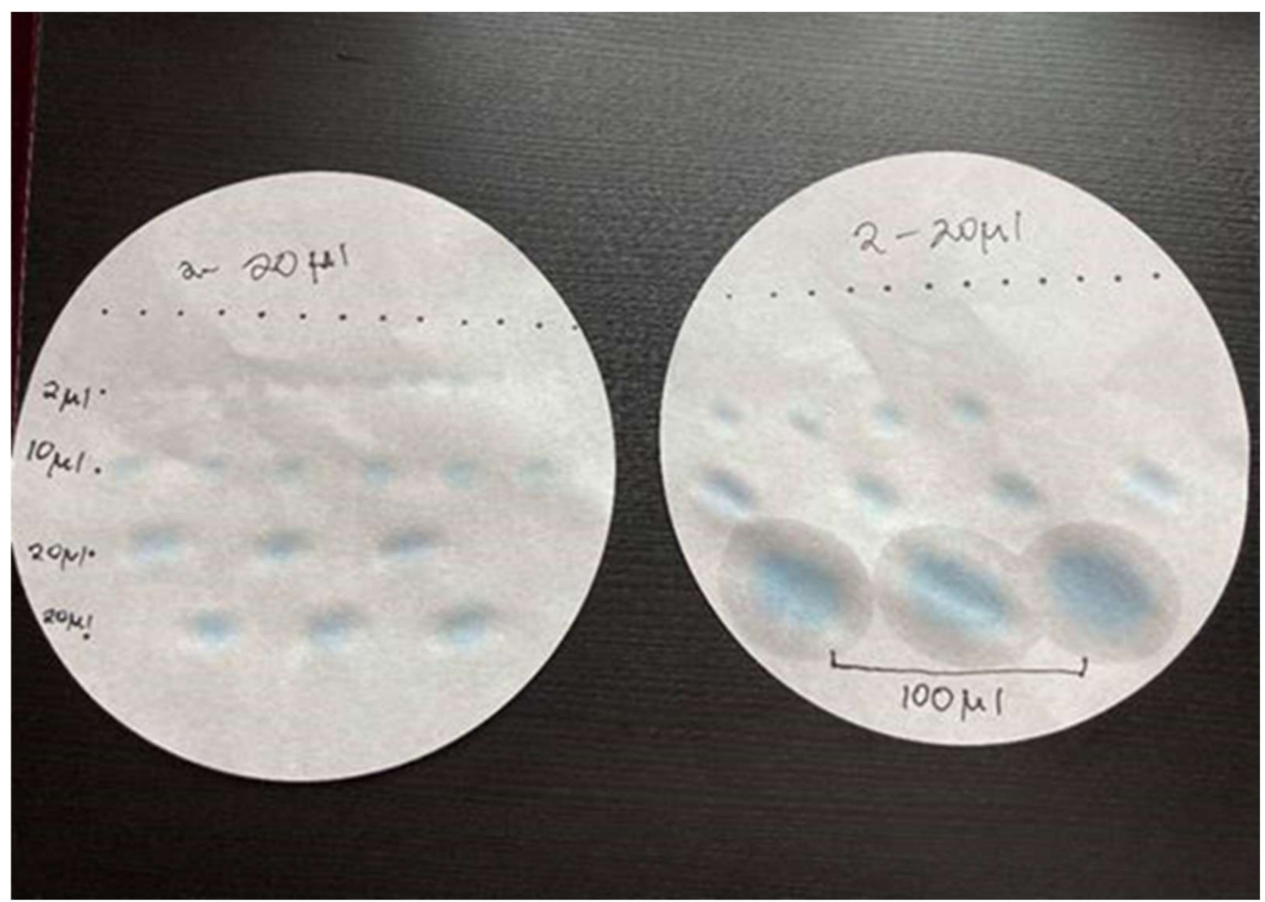

Figure 5. Example of the type of results expected in a filter paper experiment designed to enable students to test the reproducibility of their pipetting skills by measuring the size of spots produced from pipetting multiple replicates of specific volumes.

Of the students who were able to attend the practical skills assessment session at the end of the first term, $98.2 \%$ successfully completed the pipetting learning outcome at the first opportunity. This was similar to the percentage of students who had attended the assessment in previous years: $95.4 \%$ of students successfully passed the pipetting part of the assessment in 2018-2019 and the pass rate in 2019-2020 was 99.4\%.

A reflection on undertaking the pipetting tasks in the Bioskills at home kit provided by one of the first-year students is shown in the quote below. In this, the student refers to the practical skills assessment that they undertake in one of their term one modules (the practical techniques module). In this context, the student described that undertaking the exercise prior to assessment helped them to improve the consistency of their pipetting, which aided them in completing their assessment.

"I decided to do this activity right before my Practical Techniques assessment. In a previous lab, I made some errors in dilutions which affected my results. I was not as used to making dilutions in small volumes, so this activity gave me a lot of practice. Particularly, in the 20 $\mu L$ to $200 \mu L$ range. The activity also helped my pipetting become more consistent which was important to my assessment as I had to aspirate and dispense the same volume many times. As a result, I was able to complete my assessment with ease."

\subsection{Microbial Growth}

Staff prepared a growth curve for the yeast experiment as described in the student instructions to test the outcomes that students would expect to achieve (see Figure 6): presentation of experimental data in graphical form clearly enabled students to observe the effect of adding sugar to the growth media. 
A

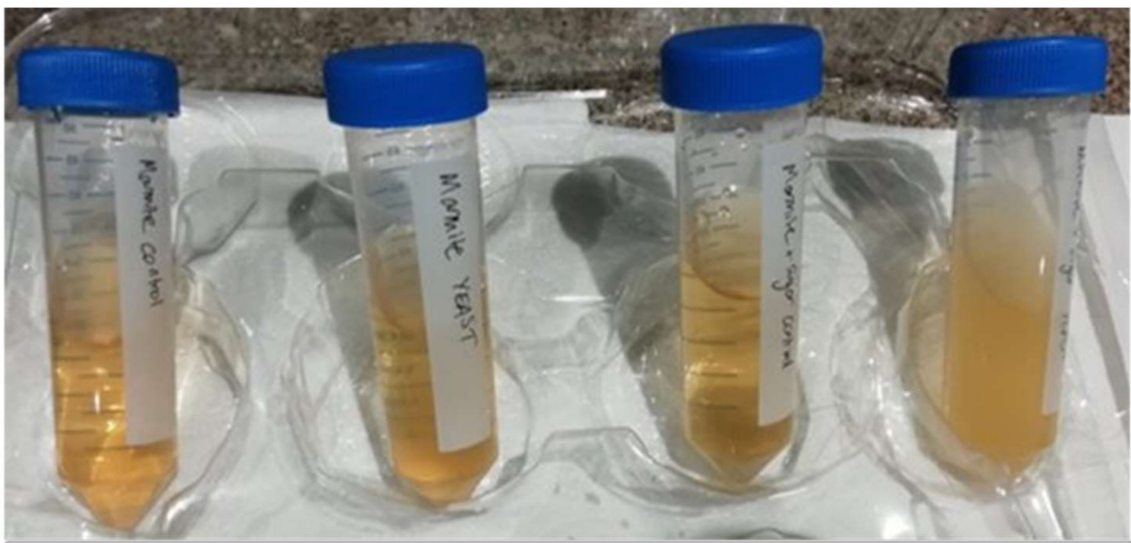

B

Preliminary growth curve test

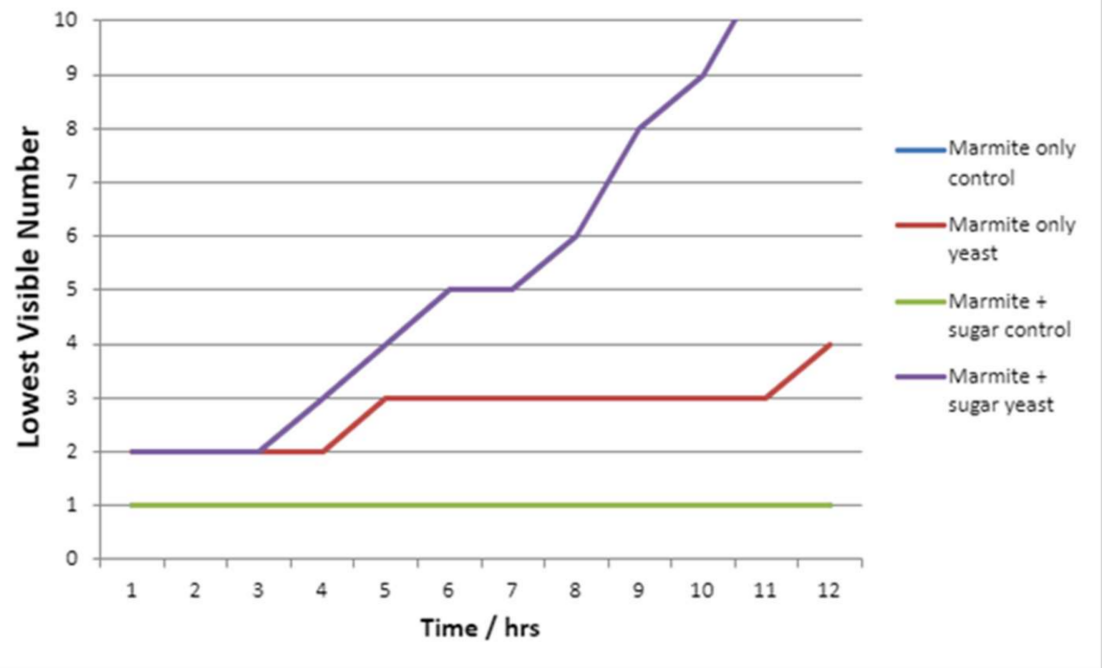

Figure 6. (A) Example of the microbial growth experiment (23 h after inoculation) conducted by staff as per the student protocol and (B) data generated from this experiment comparing growth of yeast with or without sugar added to the media over a 12-h period with appropriate experimental controls. The lines for the Marmite and sugar control are superimposed over the Marmite only control as they had the same value at each time point.

Based on comments on the discussion board, only three students confirmed that they had completed the microbial growth experiment or shared images and/or experimental data. However, the analytics from the university VLE showed that $52.4 \%$ of students engaged with the resources online.

Two students who had undertaken the microbial growth experiment reflected on the experience. For the first student, the value they ascribed to the activity was in building their confidence in pipetting, which they linked, as can be seen in the quote below, to one of the two face-to-face laboratory sessions that the students undertook. The phosphate assay laboratory class was retained as it gave students both a valuable opportunity to practice and assess the reproducibility of their pipetting, as well as being the first time they worked with small volumes in a microtiter plate (which is an assay format that many students will utilise repeatedly during their degree).

"I found doing the microbial growth curve experiment before the phosphate assay lab really useful as I felt confident with pipetting."

The quotation from the second student shows how this aspect of the Bioskills kit helped them to think more deeply about how their experiment linked to the underlying theory and also about experimental design. 
"I did this activity after I had a lecture on bacterial growth requirements. It made me think a lot about what conditions I needed to control whilst doing this experiment. For example, I tried to control temperature fluctuations by placing my tubes in a water bath near a radiator. This was to allow any heat to be distributed evenly. I also ensured that all tubes had the same amount of nutrients. As I was recording my results, it made me think about what processes were occurring inside the tube and how this affected the growth rate. I was able to use the results from this for my coursework as well. Overall, it was a very fun and useful activity to do."

\subsection{Microscopy}

The microscopy competitions ran from the start of term 1 (early October 2020) until near the end of November 2020. Within that time, students were able to submit entries to the four competitions:

- The "Tardigrade hunt": this was successfully completed by a student who identified a tardigrade approximately two weeks after the Bioskills packs were initially distributed.

- Best biological image: this was the best-supported competition with a total of five entries; an example of one of these entries can be seen in Figure 7.

- $\quad$ Best biological video: this category received three entries.

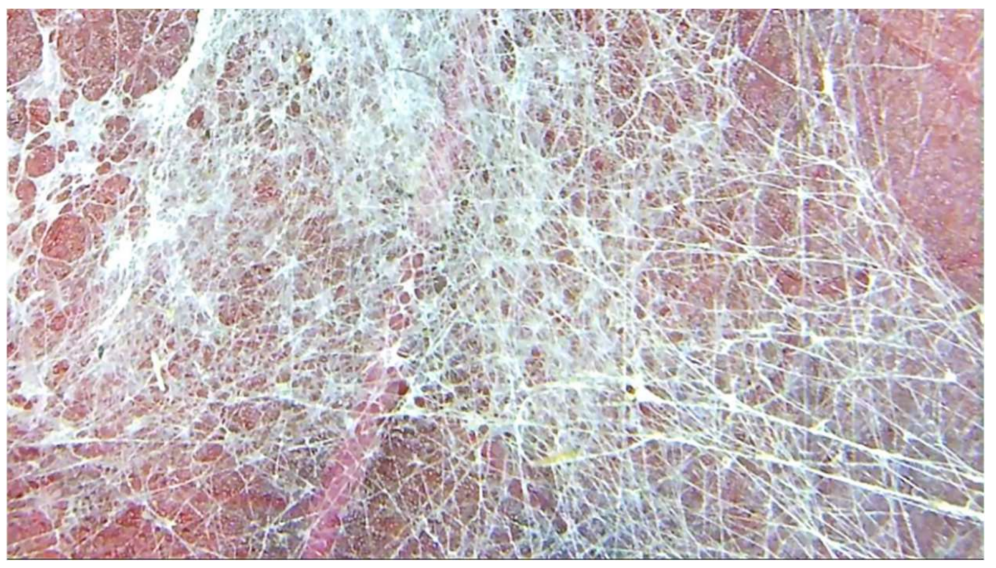

Figure 7. Image of a spider's web on the underside of a leaf taken using the Rotek digital microscope and entered into the best biological image competition.

Overall, a total of 12 competition entries were made. Whilst the number of entries was low, at least $20 \%$ of students engaged with the online resources to support setting up the microscope $(26.0 \%)$, guidance on how to use a haemocytometer $(20.4 \%)$ and information on the different competitions $(25.5 \%)$.

A similar level of engagement was seen at the celebration event, where approximately 100 students (22.2\% of the cohort) attended, with at least half of these actively voting on competition entries.

Reflecting back on the microscopy awards ceremony (which was held online due to pandemic restrictions), one of the students highlighted that, for them, this helped to bring students together. Furthermore, the competitions themselves had helped to motivate them to explore their environment, as well as positively impacting upon their subject interest (see quote below).

"... I really enjoyed it and I thought it was a fun conclusion to the competition which brought people in biosciences together for some fun in a difficult year. The competition was also a great addition as it allowed us to be creative and remember that you can learn a lot from your own immediate environment. It also encouraged me to use any equipment I have to experiment with nature around me and allow this to further my scientific understanding as well as passion." 


\section{Discussion}

\subsection{Evaluation of Bioskills at Home Initiative}

It is clear that those students who engaged with the various activities in the Bioskills at home experiments derived benefit from doing so. The quotes from students highlighted that the tasks they were set improved their confidence (particularly in terms of pipetting) and maintained or enhanced their motivation and interest in the subject. Each of these are valuable aspects of the affective domain that can have a positive impact on meaningful learning, as described by Novak [4], and which has been acknowledged by academics as an important reason for first year undergraduates to undertake practical work [16]. Our institution is not the only one to assess key practical competencies. Seery et al. [40] have used "digital badging" as a micro-accreditation to show when students had reached a certain level of skill or demonstrated competency in a particular technique. Using a digital format allows students to use these via professional networking sites which has the potential to enhance employability and may inherently motivate students to want to "collect" more badges in a similar way to computer game achievements/badges.

\subsubsection{Pipetting Task}

Building student confidence in pipetting was a significant goal, as it is a skill that most students will use throughout their course and beyond, which is why it forms part of the skills competencies that are tested in the laboratory assessment at the end of the first term. Despite reduced opportunities to practice lab skills on campus (which could be further reduced if students were self-isolating or subject to travel restrictions from their home country), the fact that the proportion of students that successfully completed the pipetting assessment is similar to that seen in the previous two years suggests that the initiative was successful in ensuring students' pipetting competencies. Pipetting was a skill that formed part of both retained laboratory classes; therefore, it is possible that students who were able to attend at least one of the laboratory classes were able to pass this assessment without the use of the online pipetting resources. This may be an explanation why the guidance on "how to operate the pipettes to dispense liquid" was the most used resource $(19.6 \%)$, as this would be the key resource for students unable to attend laboratory classes as well as students wanting to remind themselves of what they learnt in the class and practice independently. This could explain the apparent disparity between the proportion of students using the resources and the pipetting assessment success rate. For those students who made use of the pipetting experiments (up to one fifth of the student cohort), performing the pipetting experiments would have provided potential benefits in being able to improve their pipetting reproducibility and troubleshoot issues in pipetting in addition to being able to accurately use a micropipette.

\subsubsection{Bacterial Growth Curve}

In the student quote from the microbial growth experiment, there is reference to how they had considered a range of factors that were involved in the experiment and how to ensure that they controlled the conditions that they were using. This description indicates that the student was using higher order skills than would normally be expected at this level, although this had formed part of the overall aim of the task and is in keeping with the observed benefits of enquiry-based learning $[9,10]$. In the laboratory framework proposed by Seery et al. [41] in chemistry, the first year of laboratory classes have been described as important in forming a foundation in the key procedures and skill competencies that students would need to be familiar with in their discipline; a point supported by bioscience academics [16]. According to Seery et al. [41], knowledge of these procedures would be built on in subsequent years to enable students to work toward designing their own experiments, initially based on the key procedure they have learnt about but expanding on this to be able to perform open-ended experiments. Seery's proposed framework situates these higher-level skills as appropriate for the third year of Scottish undergraduate degree programmes (equivalent to a second-year undergraduate programme in England). Since 
the Bioskills activities described were undertaken by students in the first term of their first year at university, this suggests that engaging with the activities starts the process of developing higher order skills as an earlier stage than otherwise might be expected.

One area that none of the quoted students mentioned but which had been factored into the design of activities were the analytical aspects of the tasks. It is difficult to assess whether this is because the students did not perform this part of the task or whether they considered other aspects more valuable and therefore noteworthy in their reflections. This aspect was included in the study design as it is well established that undergraduate students suffer with maths anxiety and so building up students' confidence with the types of analyses that they would use on their course was considered a valuable opportunity by the academic team. An example of the extent to which science undergraduates experience maths anxiety can be seen in a study of 1153 university students in the UK. Using the maths anxiety scale (survey), it was found that maths anxiety in science disciplines (excluding computing and engineering) was the second highest amongst undergraduates with only arts, media and design students scoring more highly [42]. In particular, bioscience undergraduate students have been observed to have difficulty working with contextualised questions [43]. This is problematic as these types of questions have relevance in the context of their degree programme.

Of all the experiments designed for the Bioskills at home kit, this experiment received the most attention from students in terms of accessing the associated resources: twice as many students accessed the microbial growth curve resources (52.4\%) compared to either of the other experiments (maximum of $26.0 \%$ for microscopy resources, $22.9 \%$ for pipetting resources). Whilst it is not possible to comment on what proportion of students who accessed the material actually performed the experiment, completion of the experiment would have given experience of data analysis, graph plotting that would have complimented lecture content as well as valuable practice at experimental design (including the use of relevant controls).

\subsubsection{Microscopy}

The most significant issue that was noted in the Bioskills at home initiative was a lack of engagement with some of the activities. As can be seen in the findings, the discussion boards had relatively few contributions from students and even the most well supported areas of the boards, such as the microscopy competition, showed a relatively low number of entries (a total of 12 entries across the 4 competitions) considering that the cohort was approximately 450 students.

The original aim of the microscopy experiments in the Bioskills kit was to engage student's scientific interest and build a sense of community through the discussion boards. As described with both the pipetting and microbial growth curve, students appeared reluctant to use the boards despite the possibility of posting anonymously. However, the celebration event where students voted for winners in the microscopy competition was well supported and students attending this (which accounted for approximately a quarter of the cohort) engaged well with the session. This is supported by the student quote which highlighted that it had been a good way to bring people together.

\subsection{Technical Challenges}

The enormity of the task presented by sourcing, packing and distributing the Bioskills at home packs to the anticipated cohort of 500 first year students cannot be underestimated, particularly when set against the challenges presented by delivering on-campus practical classes alongside supporting lab classes that had moved online (e.g., by recording experiments and photographing experimental results for students to use in their assessments). Furthermore, working practice for technical and administrative staff had been significantly impacted by the pandemic with social distancing in place in the labs as well as staff working in "bubbles" to mitigate against infection risk and to ensure that technical support could be sustained even if staff were required to self-isolate. The preparation of the Bioskills bags, particularly considering the increase in anticipated intake (a 20\% increase compared to expected numbers 
of students), put increasing pressure on staff to meet short deadlines. However, a combination of meticulous planning, interpersonal support and teamwork enabled staff to meet these challenges and successfully deliver the target resources to students.

\subsection{Future Development of the Bioskills at Home Initiative}

With the arrangements for the forthcoming academic year being put into place, there is an opportunity to reflect on the successes and limitations of this initiative and how this can be developed further to meet its original objectives. Even if students are largely able to return to campus teaching and lab provision is increased, there is still value to be gained from retaining and improving the Bioskills at home project. Apparent lack of engagement with some activities was the most significant factor which limited the success of this initiative. Whilst we are in the process of collecting data to better understand the students' perspective, experience within the university overall suggests that navigating a year at university during a pandemic has proven to be a significant challenge to students and that they can easily become overwhelmed. The flexibility of the Bioskills activities was designed to allow students to work at a pace that suited them; however, it seems likely that the unstructured nature of the initiative contributed to the lack of engagement as students lacked defined deadlines for the most part. Further, it seems likely that the fact that these activities were not mandatory or assessed meant that students were more likely to engage with the activities if they already felt some degree of engagement with their course, whereas those who struggled to engage with the course may not have been inspired to participate in the initiatives without a clear incentive. With this in mind, going forward, the activities retained will be actively embedded into face-to-face tutor group sessions (typically a group of 10-12 students who are enrolled in the same course) with additional contact points with tutors and student mentors [44] used to guide the activities. It is hoped that by embedding activities into the first tutorial session that students have with their personal tutors, it will help to break the ice and start to build community between students and foster engagement with the initiative that can be sustained and developed throughout their first year.

In addition to embedding the activities in a more structured way, the activities that students are asked to perform will be streamlined to retain those considered to have the highest value and therefore being most cost effective. There was certainly value in the pipetting activities both in terms of student confidence and successful completion of the practical skills assessment, so these will be retained. However, whilst the competition celebration was valuable in terms of generating interest and community building, the microscopes were an expensive component given the level of engagement with the competitions themselves. As such, the microscopy activity will not be included in the next iteration of the Bioskills kits and instead the competition and celebration will be refocussed on other activities. In addition, as students return to campus, they will have increased opportunity to use microscopes in the laboratory so there is less incentive for students to perform this with alternative equipment. The microbial growth experiment will also be retained alongside the pipetting experiment as it is both cost effective and the resources on the VLE showed the highest level of engagement of any of the activities (52.4\%) even though this has not translated to experimental data being shared to the discussion boards. Additionally, as this experiment links not only to term 1 modules but has links to other modules in first year and beyond, this was seen as a high value activity. Raising student awareness of how these activities link to other modules, including the final year project module, through discussion with staff and student mentors could raise engagement especially as some mentors will have used this year's kit and so would be able to advocate for its benefits.

\section{Conclusions}

Whilst logistically challenging, providing first year undergraduate students with a range of practical activities to complete at home can provide benefits to their learning in a pandemic and beyond by enhancing confidence in using these techniques in lab or assessment settings, as well as potentially enhancing higher order skills such as experimental 
design. To increase engagement and community building with home lab kits requires both flexibility but also scaffolding to achieve maximum impact.

Supplementary Materials: The following supporting information can be downloaded at: https: / / www.mdpi.com/article/10.3390/educsci12020106/s1, Table S1: Skills tracker. Excerpt from the skills tracker which is being used to map the skills gained from different modules for each course for each year of study. The table below shows the modules taken by first year undergraduate students studying on the biochemistry and microbiology course. The term 1 modules (Practical Techniques in Biology and Living systems) were those used to assess what skills would be lost due to social distancing and hence one of the important considerations in deciding on the activities in the home lab kit. Skills attained from these modules are listed under different category headings: Personal, professional and reflective; generic scientific skills; subject specific skills and graduate attributes. The source of these skills ( $\mathrm{T}$ for taught as provided by lectures, seminars and lab classes; A where these are part of an assessment), which modules have these skills and the number of opportunities that there are across the different modules are shown in the skills tracker. The excerpt shown below contains examples (but not all skills) for each of the categories. The generic skills text highlighted in bold text directly link to aspects of the home labs kit.

Author Contributions: Conceptualization, J.W. and K.G.; methodology, K.G., S.R., I.K., G.M, E.S., C.R., C.N, J.T. and D.N.; validation, J.W., G.M. and S.R.; formal analysis, S.R.; investigation, J.W., K.G., S.R., I.K., L.A.D.G., E.S., C.R., C.N., M.L., J.D., J.T. and D.N.; resources, E.S.; data curation, J.D. and S.R.; writing—original draft preparation, S.R., K.G. and E.S.; writing—review and editing, J.W., K.G., S.R., M.L., C.N. and G.M.; visualization, S.R., J.W., G.M., K.G., I.K., L.A.D.G. and E.S.; project administration, J.W., K.G. and E.S. All authors have read and agreed to the published version of the manuscript.

Funding: This research received no external funding.

Institutional Review Board Statement: Not applicable.

Informed Consent Statement: Student microscopy images and reflections reproduced with permission.

Data Availability Statement: Not applicable.

Acknowledgments: The authors would like to thank the following members of the Nottingham Trent University School of Science and Technology technical and administration teams for their hard work and dedication in ensuring that the bioskills at home initiative was successfully de-livered: Ray Workman, Emma Rubbi, Laura King, Rebecca Coxhill, Ava McMullin, Shaff Hussain, James Kelly, David Green, David Wilkinson, Jayne Spence, Georgina Bradshaw, Emma Storey, Tom Brookes, Sam Poole, Macsen Fryer, Helen Wallis, Gareth Williams, Jessica Fountain, Jill McElvaney, James Kelly, Caroline Simiyu, Sowmya Suresh Menon, Luke Russell, Abby Whiley, Sam Coggin, Lisa Appleby, Tom Haffenden and Iain Walker.

Conflicts of Interest: The authors declare no conflict of interest.

\section{References}

1. Pike, N. The experimental sciences. In A Handbook for Teaching and Learning in Higher Education: Enhancing Academic Practice, 4th ed.; Routledge: Abingdon-on-Thames, Oxfordshire, UK, 2015.

2. $\quad$ Kerr, J.F.; Boulind, H.F.; Rolls, M.J. Practical Work in School Science: An Account of an Inquiry ... Into the Nature and Purpose of Practical Work in School Science Teaching in England and Wales; Routledge: Abingdon-on-Thames, Oxfordshire, UK, 1963.

3. Esparza, D.; Wagler, A.E.; Olimpo, J.T. Characterization of Instructor and Student Behaviors in CURE and Non-CURE Learning Environments: Impacts on Student Motivation, Science Identity Development, and Perceptions of the Laboratory Experience. CBE Life Sci. Educ. 2020, 19. [CrossRef]

4. Novak, J.D. Learning Theory Applied to the Biology Classroom. Am. Biol. Teach. 1980, 42, 280-285. [CrossRef]

5. Ausubel, D.P. The Psychology of Meaningful Verbal Learning; Grune \& Stratton: New York, NY, USA, 1963.

6. Bretz, S.L. Novak's Theory of Education: Human Constructivism and Meaningful Learning. J. Chem. Educ. 2001, 78, 1107. [CrossRef]

7. Galloway, K.R.; Bretz, S.L. Development of an Assessment Tool To Measure Students' Meaningful Learning in the Undergraduate Chemistry Laboratory. J. Chem. Educ. 2015, 92, 1149-1158. [CrossRef]

8. Galloway, K.R.; Bretz, S.L. Using cluster analysis to characterize meaningful learning in a first-year university chemistry laboratory course. Chem. Educ. Res. Pract. 2015, 16. [CrossRef] 
9. Hofstein, A.; Lunetta, V.N. The laboratory in science education: Foundations for the twenty-first century. Sci. Educ. 2004, 88, 28-54. [CrossRef]

10. Kahn, P.; O'Rourke, K. Understanding enquiry-based learning. In Handbook of Enquiry and Problem-based Learning; Barrett, T., Mac Labhrainn, I., Fallon, H., Eds.; CELT: Galway, Ireland, 2005; pp. 1-12.

11. Kipnis, M.; Hofstein, A. The inquiry laboratory as a source for development of metacognitive skills. Int. J. Sci. Math. Educ. 2008, 6, 601-627. [CrossRef]

12. Adams, D.J. Current Trends in Laboratory Class Teaching in University Bioscience Programmes. Biosci. Educ. 2009, 13(1), 1-14. [CrossRef]

13. Kuldell, N.H. How Golden is Silence? Teaching Undergraduates the Power and Limits of RNA Interference. CBE Life Sci. Educ. 2006, 5. [CrossRef]

14. Brauner, A.; Carey, J.; Henriksson, M.; Sunnerhagen, M.; Ehrenborg, E. Open-ended assignments and student responsibility Biochem. Mol. Biol. Educ. 2007, 35. [CrossRef]

15. Kolkhorst, F.W.; Mason, C.L.; DiPasquale, D.M.; Patterson, P.; Buono, M.J. An inquiry-based learning model for an exercise physiology laboratory course. Adv. Physiol. Educ. 2011, 25. [CrossRef]

16. Adams, D.; Arkle, S.; Bevan, R.; Boachie-Ansah, G.; Bradshaw, T.; Cameron, G.; Campbell, A.M.; Chamberlain, M.; Gibson, A.; Gowers, D.; et al. 1st Year practicals: Their Role in Developing Future Bioscientists; HEA Centre for Bioscience Report; Centre for Bioscience: Leeds, UK, 2008. Available online: https://synergy.st-andrews.ac.uk/vannesmithlab/files/2015/08/Adams_et_al0 8CentreBioReport.pdf (accessed on 23 June 2021).

17. Carnduff, J.; Reid, N. Enhancing Undergraduate Chemistry Laboratories: Pre-Laboratory and Post-Laboratory Exercises; Royal Society of Chemistry: London, UK, 2003.

18. Johnstone, A.H.; Al-Shuaili, A. Learning in the laboratory; some thoughts from the literature. Univ. Chem. Educ. $2001,5,42-51$.

19. QAA. Subject Benchmark Statement: Biosciences. The Quality Assurance Agency for UK Higher Education, Oct. 2019. Available online: https://www.qaa.ac.uk/docs/qaa/subject-benchmark-statements/subject-benchmark-statement-biosciences.pdf (accessed on 23 June 2021)

20. Department for Education. Guidance Higher Education Providers: Coronavirus (COVID-19). Available online: https://www.gov. uk/government/publications/higher-education-reopening-buildings-and-campuses (accessed on 26 July 2021).

21. Kirk, S.; Cosgrove, M.; Baker, D.; Ward, A.; Richards, A. T-enabled bioscience and chemistry teaching in Nottingham Trent University's Rosalind Franklin building. In Laboratories for the 21st century in STEM higher education: A compendium of current UK practice and an insight into future directions for laboratory-based teaching and learning; Loughborough University: Loughborough, UK, 2013. Available online: https:/ / hdl.handle.net/2134/13389 (accessed on 23 June 2021).

22. Coward, K.; Gray, J. Audit of Practical work undertaken by undergraduate bioscience students across the UK higher education sector; Royal Society of Biology: London, UK, 2014. Available online: https:/ /www.rsb.org.uk/images/SB/UG-Practical-Work-Report-Web. pdf (accessed on 23 June 2021).

23. Royal Society of Biology. Degree Accreditation Programme. 2021. Available online: https://www.rsb.org.uk/education/ accreditation/employers (accessed on 14 July 2021).

24. Sotomayor-Moriano, J.; Pérez-Zúñiga, G.; Soto, M. A virtual laboratory environment for control design of a multivariable process. IFAC PapersOnLine 2019, 52, 15-20. [CrossRef]

25. Lewis, D.I. The Pedagogical Benefits and Pitfalls of Virtual Tools for Teaching and Learning Laboratory Practices in the Biological Sciences; The Higher Education Academy-STEM: Heslington, York, UK, 2014.

26. Gibbons, N.J.; Evans, C.; Payne, A.; Shah, K.; Griffin, D.K. Computer Simulations Improve University Instructional Laboratories. Cell Biol. Educ. 2004, 3. [CrossRef] [PubMed]

27. Gregory, S.-J.; di Trapani, G. A blended learning approach to laboratory preparation. Int. J. Innov. Sci. Math. Educ. 2012, 20,1. Available online: https:/ / openjournals.library.sydney.edu.au/index.php/CAL/article/view/6650 (accessed on 23 June 2021).

28. Coleman, S.K.; Smith, C.L. Evaluating the benefits of virtual training for bioscience students. High. Educ. Pedagog. 2019, 4, 287-299. [CrossRef]

29. Dyrberg, N.R.; Treusch, A.H.; Wiegand, C. Virtual laboratories in science education: Students' motivation and experiences in two tertiary biology courses. J. Biol. Educ. 2017, 51, 358-374. [CrossRef]

30. Rodgers, T.L.; Cheema, N.; Vasanth, S.; Jamshed, A.; Alfutimie, A.; Scully, P.J. Developing pre-laboratory videos for enhancing student preparedness. Eur. J. Eng. Educ. 2020, 45, 292-304. [CrossRef]

31. Francis, N. It's a Brave New (Educational) world. Advanced HE, September 2020. Available online: https://www.advance-he.ac. uk/news-and-views/its-brave-new-educational-world (accessed on 30 June 2021).

32. Rushworth, J.; Moore, T.J.; Rogoyski, B. LectureRemotely. 2021. Available online: https:/ /www.lecturemotely.com/ (accessed on 14 July 2021).

33. Lam, R. What students do when encountering failure in collaborative tasks. npj Sci. Learn. 2019, 4. [CrossRef] [PubMed]

34. Vygotsky, L.S. Thought and Language; The MIT Press: Cambridge, MA, USA, 1986.

35. Croft, N.; Dalton, A.; Grant, M. Overcoming Isolation in Distance Learning: Building a Learning Community through Time and Space. J. Educ. Built Environ. 2010, 5. [CrossRef]

36. Kaufmann, R.; Vallade, J.I. Exploring connections in the online learning environment: Student perceptions of rapport, climate, and loneliness. Interact. Learn. Environ. 2020. [CrossRef] 
37. Reedy, A.K. Rethinking online learning design to enhance the experiences of Indigenous higher education students. Australas. J. Educ. Technol. 2019, 35. [CrossRef]

38. Wolf, J. COC: “Introduction to Biotechnology” Custom Lab Exercises. 2019. Available online: https://www.canyons.edu/ _resources/documents/academics/biology/biotech_resources/lab_files/PipetingAccuracyPrecision6-28-12.pdf (accessed on 21 January 2022).

39. Tankeshwar, A. Preparation of McFarland Turbidity Standards. 2021. Available online: https://microbeonline.com/ preparationmcfarland-turbidity-standards/ (accessed on 1 September 2021).

40. Seery, M.K.; Agustian, H.Y.; Doidge, E.D.; Kucharski, M.M.; O’Connor, H.M.; Price, A. Developing laboratory skills by incorporating peer-review and digital badges. Chem. Educ. Res. Pract. 2017, 18. [CrossRef]

41. Seery, M.K.; Agustian, H.Y.; Zhang, X. A Framework for Learning in the Chemistry Laboratory. Isr. J. Chem. 2019, 59, 546-553. [CrossRef]

42. Hunt, T.E.; Clark-Carter, D.; Sheffield, D. The Development and Part Validation of a U.K. Scale for Mathematics Anxiety. J. Psychoeduc. Assess. 2011, 29. [CrossRef]

43. Tariq, V.N. Defining the problem: Mathematical errors and misconceptions exhibited by first-year bioscience undergraduates. Int. J. Math. Educ. Sci. Technol. 2008, 39. [CrossRef]

44. Nottingham Trent University, “CERT Student Mentors,". 2021. Available online: https://www.ntu.ac.uk/c/censce/opportunitiesfor-students / cert-mentoring (accessed on 30 July 2021). 